This is the accepted version of the article:

Espín J., Garzón-Tovar L., Boix G., Imaz I., Maspoch D.. The photothermal effect in MOFs: Covalent post-synthetic modification of MOFs mediated by UV-Vis light under solvent-free conditions. Chemical Communications, (2018). 54. : 4184 - . 10.1039/c8cc01593g.

Available at: https://dx.doi.org/10.1039/c8cc01593g 


\title{
The Photothermal Effect in MOFs: Covalent Post-Synthetic Modification of MOFs Mediated by UV-Vis Light under Solvent-Free Conditionst
}

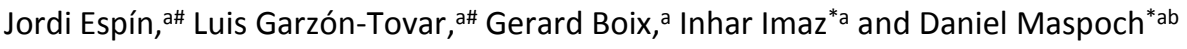 \\ a. Catalan Institute of Nanoscience and Nanotechnology (ICN2), CSIC and The Barcelona Institute of Science and Technology, Campus UAB, Bellaterra, \\ 08193 Barcelona, Spain. E-mail: daniel.maspoch@icn2.cat \\ b. ICREA, Pg. Lluís Companys 23, 08010 Barcelona, Spain \\ \# Both authors contributed equally
}

Here, we report the covalent post-synthetic modification (CPSM) of MOFs using the photothermal effect. Specifically, we subjected mixtures of a photothermally active MOF and another reagent to irradiation with a UV-Vis lamp. This caused the MOF to heat up, which in turn caused the other reagent to melt and subsequently react with the functional groups on the walls of the MOF pores. We have exploited this dual function of MOFs as both heater and host for CPSMs to achieve rapid formation of amides from the reaction of representative MOFs (UiO-66-NH2 or MIL-101-NH2-(AI)) with anhydrides under solvent-free conditions. In addition, this approach enables more complex CPSMs in MOFs such as the formation of amides in UiO-66- $\mathrm{NH}_{2}$ by using an aldehyde through a cascade reaction.

Photo-irradiation of certain materials causes them to heat up. This is known as the photothermal effect. Ideally, one could exploit this conversion of light into heat to selectively increase the local temperature of a given material and its surroundings, while minimizing heat diffusion and energy loss. Photothermal materials have been harnassed for myriad applications such as cancer theranostics ${ }^{1-7}$ and in water evaporation, ${ }^{8-10}$ sterilisation ${ }^{11}$ or desalination ${ }^{12,13}$ devices. Such materials include organometallic complexes, ${ }^{14}$ polymers, ${ }^{1,2}, 15$ carbon-based materials, ${ }^{8,12,}{ }^{16}$ noble-metal and hybrid nanoparticles, ${ }^{9-11,13,17}$ and semiconductors. ${ }^{5-7,18}$

Recently, we found that some porous metal-organic frameworks (MOFs) can exhibit a pronounced photothermal effect. After UV-Vis irradiation (300-650 nm), MOFs exhibiting adsorption bands in this range can reach temperatures exceeding $120^{\circ} \mathrm{C}$ within minutes. For example, $100 \mathrm{mg}$ and $25 \mathrm{mg}$ of the archetypical MOF UiO-66- $\mathrm{NH}_{2}$ reaches a temperature of $140{ }^{\circ} \mathrm{C}$ and $78{ }^{\circ} \mathrm{C}$, respectively, after only 5 minutes of irradiation $\left(500 \mathrm{~mW} \cdot \mathrm{cm}^{-2}\right)$ at a light guide-to-sample distance of $7 \mathrm{~cm}$ (Figure S1, ESI ${ }^{\dagger}$ ). We demonstrated that this localised heat can be used to efficiently remove trapped and coordinated solvent molecules from within the MOF structure to generate activated MOFs at atmospheric pressure in unprecedented short times. ${ }^{19}$

Covalent post-synthetic modifications (CPSMs) are employed to derivatise MOFs with diverse functional groups, which enables tailoring of physicochemical properties (e.g. wettability ${ }^{20,21}$ ) and of functions (e.g. catalysis ${ }^{22,}{ }^{23}$ and gas sorption). ${ }^{24}$ These modifications are generally done by reacting MOF crystals with other reagents in solution for long periods of time (from hours to days). ${ }^{25}$ However, alternative methods have recently been proposed to reduce the solvent volume and/or the reaction time. For example, our group has shown that Schiff-base condensation reactions between aldehydes and amines inside MOFs can be performed in mere seconds, by using spray-drying. ${ }^{26}$ The first reported solvent-free method for the post-synthetic modification of MOFs was vapour diffusion. Thus, Ranocchiari, Bokhoven et al. described the CPSM of solid amino-tagged MOFs by exposing them to vapours produced by heating liquid aldehydes or anhydrides at $120^{\circ} \mathrm{C}$ under vacuum for $16 \mathrm{~h} .{ }^{27}$ More recently, Wuttke et al. reported a second solvent-free CPSM strategy based on the direct heating of a solid mixture of an amino-terminated MOF and a carboxylic acid derivative (acid anhydride, acid chloride or carboxylic acid) at ca. $100{ }^{\circ} \mathrm{C}$ for minutes. ${ }^{28}$ Similarly, Richardson et al. demonstrated that 
a Newman-Kwart rearrangement on a dimethylthiocarbamate-functionalized IRMOF-9 occurs by direct heating it at $285^{\circ} \mathrm{C} .{ }^{29}$

In the study reported here, we extended our previous work on localised photothermal activation of MOFs to perform CPSMs of MOFs under solvent-free conditions. We show that MOFs, once mixed with another reagent and irradiated by a UV-Vis lamp, can simultaneously act as both heater and host for CPSMs. The MOF crystals reach high temperatures, thereby heating their surroundings and melting the other reagent, which then reacts with the functional groups of the MOF. As a final step of this process, the mixture is washed with a solvent to remove the unreacted chemical reagent, and the post-modified MOF is collected by centrifugation and dried. Three conditions must be met in this CPSM approach. Firstly, the MOF must have functional groups available for reaction. Secondly, the MOF must be phothermally active. And finally, the chemical reagent must melt at a temperature below that of the previously heated MOF.

To demonstrate the feasibility of this solvent-free, photo-induced CPSM method, we chose UiO-66- $\mathrm{NH}_{2}$ as a representative MOF, as it is strongly photothermal (vide supra) and contains free amino groups available for derivatization. As a model reaction, we reacted this MOF with anhydrides to form amides. Thus, UiO-66- $\mathrm{NH}_{2}$ was synthesised and activated under previously reported conditions, and obtained as a pure phase as confirmed by X-ray powder diffraction (XRPD) and $\mathrm{N}_{2}$ adsorption measurements ( $\mathrm{S}_{\text {BET }}$ value: $936 \mathrm{~m}^{2} \mathrm{~g}^{-1}$; Figs. S2 and S3, ESI $\left.{ }^{+}\right)^{30}$ Once synthesised, the activated UiO-66- $\mathrm{NH}_{2}(25 \mathrm{mg})$ was mixed with maleic anhydride $(\mathrm{MA})$ (melting point: $51-56{ }^{\circ} \mathrm{C}$ ) at molar ratios (2-aminoterephtalate/MA) of 1:1, 1:3 and 1:6. Each solid mixture was placed between two quartz slides (Scheme 1). A light guide was fixed at a distance of $7 \mathrm{~cm}$ from the sample. Each pair of slides was then irradiated (irradiance: $500 \mathrm{~mW} \cdot \mathrm{cm}^{-2}$ ) for $30 \mathrm{~min}$, causing the MOF to reach a temperature of $78-82^{\circ} \mathrm{C}$ (Fig. S4 and S5, ESI†). To ensure homogeneous light exposure, each slide pair was turned around after 15 minutes. Note that, as a control experiment, a solid sample of MA alone was irradiated for 30 minutes; it did not exhibit any temperature increase. Finally, the resulting irradiated mixtures were washed with DMF and acetone and dried at room temperature. The different samples were named as (UiO-66-MA) anhydride.

\section{--- SCHEME 1 and FIGURE 1 ---}

Using XRPD, we confirmed that all three samples retained the crystallinity of the starting UiO66- $\mathrm{NH}_{2}$ (Figs. $1 \mathrm{~b}$ and $\mathrm{S6}, \mathrm{ESI}+$ ). To determine the degree of post-synthetic conversion, the digested powders ( $5 \% \mathrm{HF} / \mathrm{DMSO}-d_{6}$ ) were analysed by ${ }^{1} \mathrm{H}-\mathrm{NMR}$ spectroscopy. The conversion rates were then calculated by comparing the integration of the peak corresponding to unmodified 2-aminoterephtalic acid (7.34 ppm) to that of the peak corresponding to the alkenyl hydrogens of the newly formed amide moiety $(6.58 \mathrm{ppm})$. The conversion rates were $69.9 \% \pm 0.4 \%$ for $(\mathrm{UiO}-66-\mathrm{MA})_{1}, 85.1 \% \pm 1.0 \%$ for (UiO$66-\mathrm{MA})_{3}$ and $83.4 \% \pm 0.3 \%$ for UiO-66-MA) ${ }_{6}$ (Figs. $1 \mathrm{a}$ and $\mathrm{S7}, \mathrm{ESI}+$ ). These values indicated that increasing the number of equivalents of MA from three to six did not provide a significant increase in product.

Having determined the optimal molar ratio of $\mathrm{NH}_{2}$-bdc/MA to be 1:3, we then decided to study the effects of using a stronger irradiation intensity or a longer reaction time on a mixture of photoactivated UiO-66- $\mathrm{NH}_{2}$ and MA. To assess the intensity, we used $900 \mathrm{~mW} \cdot \mathrm{cm}^{-2}$ (instead of $500 \mathrm{~mW} \cdot \mathrm{cm}^{-}$ 2 , as above), which we attained by setting the distance between the light-guide and the sample to 5 $\mathrm{cm}$ (instead of $7 \mathrm{~cm}$, as above). To study the reaction time, we irradiated the mixture for $60 \mathrm{~min}$ (instead of $30 \mathrm{~min}$, as above). As confirmed by XRPD, neither of these more aggressive conditions altered the structure of the parent $\mathrm{UiO}-66-\mathrm{NH}_{2}$ (Fig. S8 and S9, ESI + ). In these reactions, the post- 
synthetic conversions were $78.5 \% \pm 1.5 \%$ for the reaction done at a distance of $5 \mathrm{~cm}$ and $83.2 \% \pm 4.9 \%$ for the reaction irradiation at $60 \mathrm{~min}$ (Fig. S10, ESI + ). These values were similar (or even lower) to those obtained under the original (milder) conditions. Thus, for optimal reaction conditions, we chose a $\mathrm{NH}_{2}$-bdc/MA molar ratio of 1:3; a light guide-to-sample distance of $7 \mathrm{~cm}\left(500 \mathrm{~mW} \cdot \mathrm{cm}^{-2}\right)$; and an irradiation time of $30 \mathrm{~min}$.

We found further evidence of amide formation by electrospray-ionisation mass spectrometry (ESI-MS) of the digested (UiO-66-MA) $)_{3}$, which showed a peak at $m / z=278.0$ that matched the molecular formula of the desired amide product $\left[\mathrm{C}_{12} \mathrm{H}_{8} \mathrm{NO}_{7}\right]^{-}(\mathrm{m} / z=278.0)$ (Fig S11, ESI+). Finally, the $\mathrm{N}_{2}$ sorption isotherm of (UiO-66-MA) ${ }_{3}$ measured at $77 \mathrm{~K}$ showed an $\mathrm{S}_{\text {ВET }}$ value of $699 \mathrm{~m}^{2} \mathrm{~g}^{-1}$, confirming that the product MOF had a smaller surface area than the starting MOF, as expected for formation of amide groups, which are sterically bulkier than amino groups (Fig. 1c). ${ }^{31}$

We then applied our optimised conditions to the reaction of UiO-66- $\mathrm{NH}_{2}$ with benzoic anhydride (BA) (melting point: $38-42{ }^{\circ} \mathrm{C}$ ). Thus, a solid mixture of UiO-66- $\mathrm{NH}_{2}(25 \mathrm{mg})$ and $\mathrm{BA}\left(\mathrm{NH}_{2}-\right.$ bdc:BA molar ratio of 1:3) was irradiated at $500 \mathrm{~mW} \cdot \mathrm{cm}^{-2}$ for $30 \mathrm{~min}$, reaching a temperature of 78-82 ${ }^{\circ} \mathrm{C}$ (Fig. S12 and S13, ESI ${ }^{+}$). The resulting (UiO-66-BA) ${ }_{3}$ was first analysed by XRPD, which confirmed that it had retained the crystallinity of the parent MOF (Fig. 1b). As above, the formation of the expected amide group was corroborated by ESI-MS, in which the peak at $m / z=284.1$ matched the molecular formula of the product $\left[\mathrm{C}_{15} \mathrm{H}_{10} \mathrm{NO}_{5}\right]^{-}(\mathrm{m} / z=284.1)$ (Fig. S14, ESI $\mathrm{f}$ ). Also, as above, the formation of these amide groups led to a smaller surface area in the final MOF $\left(\mathrm{S}_{\text {BET }}: 621 \mathrm{~m}^{2} \mathrm{~g}^{-1}\right)$ (Fig. 1c). ${ }^{28}$ In this case, comparing the integration of the ${ }^{1} \mathrm{H}$-NMR peaks corresponding to unmodified 2 aminoterephtalic acid $(7.34 \mathrm{ppm})$ and each amide moiety $(8.14 \mathrm{ppm})$ revealed a conversion rate of $48.6 \% \pm 1.0 \%$ (Fig. 1a). We attributed this lower conversion (relative to that for the reaction with MA) to the bulky phenyl substituents of $B A$, which may have slowed down diffusion of the reagent into the MOF pore channels.

To demonstrate the versatility of our CPSM method, we chose to study a second amino-tagged MOF: MIL-101- $\mathrm{NH}_{2}$-(Al). This MOF is also photothermally active: when irradiated for $30 \mathrm{~min}$ at 265 $\mathrm{mW} \cdot \mathrm{cm}^{-2}$ (light guide-to-sample distance of $9 \mathrm{~cm}$ ), it reaches a temperature of $61^{\circ} \mathrm{C}$; and when irradiated at $500 \mathrm{~mW} \cdot \mathrm{cm}^{-2}$, reaches $72{ }^{\circ} \mathrm{C}$ (Fig S15, ESI†). Thus, MIL-101- $\mathrm{NH}_{2}$-(Al) was first synthesised under solvothermal conditions, which afforded it as a pure phase, as confirmed by XRPD and $\mathrm{N}_{2}$ sorption measurements $\left(\mathrm{S}_{\mathrm{BET}}=2702 \mathrm{~m}^{2} \mathrm{~g}^{-1}\right)$ (Fig. S16 and S17, ESIT). ${ }^{28}$ Then, this MOF $(25 \mathrm{mg}$ ) was reacted with either MA, under the optimised conditions for UiO-66- $\mathrm{NH}_{2}$ (Fig. S18 and 19, ESI+), or BA, using the lower irradiance intensity of $265 \mathrm{~mW} \cdot \mathrm{cm}^{-2}$ (Fig. S22 and S23, ESI + ). A lower intensity was used for the BA reaction because in initial tests, the value of $500 \mathrm{~mW} \cdot \mathrm{cm}^{-2}$ led to a decrease in the crystallinity of MIL-101- $\mathrm{NH}_{2}$-(Al) due to a post-synthetic ligand exchange (62.9 $\pm 1.8 \%$ ) between the $\mathrm{NH}_{2}$-bdc ligands and benzoic acid molecules formed during the amide formation (Fig. S24 and S25, ESI + ). The resulting (MIL-101-(AI)-MA) $)_{3}$ and (MIL-101-(AI)-BA) ${ }_{3}$ were analysed by XRPD and ESI-MS, which also confirmed that they each retained the crystallinity of the parent MOF (Fig. 2b) and exhibited the expected amide formation (Fig. S21 and S27, ESI + ). The conversion rates were $79.1 \% \pm$ $1.4 \%$ for (MIL-101-(AI)-MA) $)_{3}$ and $44 \% \pm 2.5 \%$ for (MIL-101-(AI)-BA) ${ }_{3}$ (Fig S20 and S26, ESI†). These results were consistent with the gradual decrease in surface area in each case, as indicated by the corresponding $\mathrm{S}_{\mathrm{BET}}$ values (determined by the $\mathrm{N}_{2}$-sorption isotherms at $77 \mathrm{~K}$ ): $549 \mathrm{~m}^{2} \mathrm{~g}^{-1}$ for (MIL-101(Al)-MA) ${ }_{3}$ and $774 \mathrm{~m}^{2} \mathrm{~g}^{-1}$ for (MIL-101-(Al)-BA) ${ }_{3}$ (Fig. 2b).

\section{--- FIGURE 2 and Table 1---}

The possibility to perform CPSMs under UV-Vis light allows one to incorporate photochemical reactions in these processes and therefore, provide researchers with novel reactions from which to 
introduce functionalities to MOFs. For example, our CPSM approach provides a simple route towards creating an amide (rather than the expected imine) starting from an aldehyde (in this case, 4bromobenzaldehyde $(\mathrm{BrBA})$ ) through a cascade reaction (Scheme 2 ). This reaction first involves the generation of a singlet oxygen by UV-Vis light that reacts with BrBA to produce 4-bromobenzoic acid (Fig. S28 and S29, ESI+). ${ }^{32}$ Then, this carboxylic acid reacts with the amino groups of the MOF to produce the amide. CPSM through this cascade reaction was done by irradiating a solid mixture of UiO-66- $\mathrm{NH}_{2}\left(25 \mathrm{mg}\right.$ ) and BrBA ( $\mathrm{NH}_{2}$-bdc: BrBA molar ratio of 1:6) at $500 \mathrm{~mW} \cdot \mathrm{cm}^{-2}$ for $30 \mathrm{~min}$. The resulting (UiO-66-BrBA) 6 was first analyzed by XRPD, which confirmed that it had retained the crystallinity of the parent MOF (Fig. S30, ESI†). Formation of the amide group was evidenced by ESIMS, from which the peak at $m / z=362.0$ matched with the molecular formula of the amide product $\left[\mathrm{C}_{15} \mathrm{H}_{10} \mathrm{BrNO}_{5}\right]^{-}(\mathrm{m} / \mathrm{z}=362.0)$ (Fig. S31, ESI). The conversion rate of this photo-induced cascade reaction was of $54.7 \% \pm 3.4 \%$ (Fig. S32, ESI).

\section{--- SCHEME 2 ---}

In summary, we have shown that the photothermal effect in MOFs can be exploited for their CPSM in the solid state. Use of MOFs as photo-activated heaters should be amenable to performing other functions, such as triggering the release of species adsorbed in MOFs; confining reactions to the inside of MOF pores or to the crystal surfaces of MOFs; and increasing the efficiency of MOF catalysts.

This work was supported by the EU FP7 ERC-Co 615954, the Spanish MINECO (project PN MAT2015-65354-C2-1-R), and the Catalan AGAUR (project 2014 SGR 80). It was also funded by the CERCA Programme/Generalitat de Catalunya. ICN2 acknowledges the support of the Spanish MINECO through the Severo Ochoa Centers of Excellence Programme and the project MAT2016-77608-C3-1P, under Grant SEV-2013-0295. J.E. acknowledges the MINECO for the FPI fellowship.

\section{Conflicts of interest}

There are no conflicts to declare.

\section{Notes and references}

1. K. Yang, H. Xu, L. Cheng, C. Sun, J. Wang and Z. Liu, Adv. Mater., 2012, 24, 5586-5592.

2. L. Xu, L. Cheng, C. Wang, R. Peng and Z. Liu, Polym. Chem., 2014, 5, 1573-1580.

3. S. Li, X. Wang, R. Hu, H. Chen, M. Li, J. Wang, Y. Wang, L. Liu, F. Lv, X.-J. Liang and S. Wang, Chem. Mater., 2016, 28, 8669-8675.

4. C. Loo, A. Lowery, N. Halas, J. West and R. Drezek, Nano Lett., 2005, 5, 709-711.

5. X. Huang, W. Zhang, G. Guan, G. Song, R. Zou and J. Hu, Acc. Chem. Res., 2017, 50, 2529-2538.

6. C. M. Hessel, V. P. Pattani, M. Rasch, M. G. Panthani, B. Koo, J. W. Tunnell and B. A. Korgel, Nano Lett., 2011, 11, 2560-2566.

7. Q. Tian, F. Jiang, R. Zou, Q. Liu, Z. Chen, M. Zhu, S. Yang, J. Wang, J. Wang and J. Hu, ACS Nano, 2011, 5, 9761-9771.

8. H. Ghasemi, G. Ni, A. M. Marconnet, J. Loomis, S. Yerci, N. Miljkovic and G. Chen, Nat. Commun., 2014, 5, 4449.

9. O. Neumann, A. S. Urban, J. Day, S. Lal, P. Nordlander and N. J. Halas, ACS Nano, 2013, 7, 4249. 
10. Y. Liu, S. Yu, R. Feng, A. Bernard, Y. Liu, Y. Zhang, H. Duan, W. Shang, P. Tao, C. Song and T. Deng, Adv. Mater., 2015, 27, 2768-2774.

11. O. Neumann, C. Feronti, A. D. Neumann, A. Dong, K. Schell, B. Lu, E. Kim, M. Quinn, S. Thompson, N. Grady, P. Nordlander, M. Oden and N. J. Halas, PNAS, 2013, 110, 11677-11681.

12. X. Li, W. Xu, M. Tang, L. Zhou, B. Zhu, S. Zhu and J. Zhu, PNAS, 2016, 113, 13953-13958.

13. L. Zhou, Y. Tan, J. Wang, W. Xu, Y. Yuan, W. Cai, S. Zhu and J. Zhu, Nat. Photonics, 2016, 10, 393.

14. X. He, X. He, S. Li, K. Zhuo, W. Qin, S. Dong, J. Chen, L. Ren, G. Liu and H. Xia, Polym. Chem., 2017, 8, 3674-3678.

15. I. H. El-Sayed, X. Huang and M. A. El-Sayed, Cancer Letters, 2006, 239, 129-135.

16. Y. Zeng, J. Yao, B. A. Horri, K. Wang, Y. Wu, D. Li and H. Wang, Energy Environ. Sci., 2011, 4, 4074-4078.

17. Z. Wang, Y. Liu, P. Tao, Q. Shen, N. Yi, F. Zhang, Q. Liu, C. Song, D. Zhang, W. Shang and T. Deng, Small, 2014, 10, 3234-3239.

18. X. Bu, D. Zhou, J. Li, X. Zhang, K. Zhang, H. Zhang and B. Yang, Langmuir, 2014, 30, 1416-1423.

19. J. Espín, L. Garzón-Tovar, A. Carné-Sánchez, I. Imaz and D. Maspoch, ACS Appl. Mater. Interfaces, 2018, 10, 9555-9562.

20. H. N. Rubin and M. M. Reynolds, Inorg. Chem., 2017, 56, 5266-5274.

21. C. Liu, Q. Liu and A. Huang, Chem. Commun., 2016, 52, 3400-3402.

22. J. Wang, M. Yang, W. Dong, Z. Jin, J. Tang, S. Fan, Y. Lu and G. Wang, Catal. Sci. Technol., 2016, 6, 161-168.

23. C. J. Doonan, W. Morris, H. Furukawa and O. M. Yaghi, J. Am. Chem. Soc., 2009, 131, 94929493.

24. J. A. Thompson, N. A. Brunelli, R. P. Lively, J. R. Johnson, C. W. Jones and S. Nair, J. Phys. Chem. C, 2013, 117, 8198-8207.

24. J. A. Thompson, N. A. Brunelli, R. P. Lively, J. R. Johnson, C. W. Jones and S. Nair, J. Phys. Chem. C, 2013, 117, 8198-8207.

25. S. M. Cohen, Chem. Rev., 2012, 112, 970-1000.

26. L. Garzón-Tovar, S. Rodríguez-Hermida, I. Imaz and D. Maspoch, J. Am. Chem. Soc., 2017, 139, 897-903.

27. M. Servalli, M. Ranocchiari and J. A. Van Bokhoven, Chem. Commun., 2012, 48, 1904-1906.

28. H. Hintz and S. Wuttke, Chem. Mater., 2014, 26, 6722-6728.

29. T. A. Ablott, M. Turzer, S. G. Telfer and C. Richardson, Cryst. Growth Des., 2016, 16, 7067-7073.

30. F. Ragon, P. Horcajada, H. Chevreau, Y. K. Hwang, U. H. Lee, S. R. Miller, T. Devic, J.-S. Chang and C. Serre, Inorg. Chem., 2014, 53, 2491-2500.

31. S. J. Garibay and S. M. Cohen, Chem. Commun., 2010, 46, 7700-7702. 


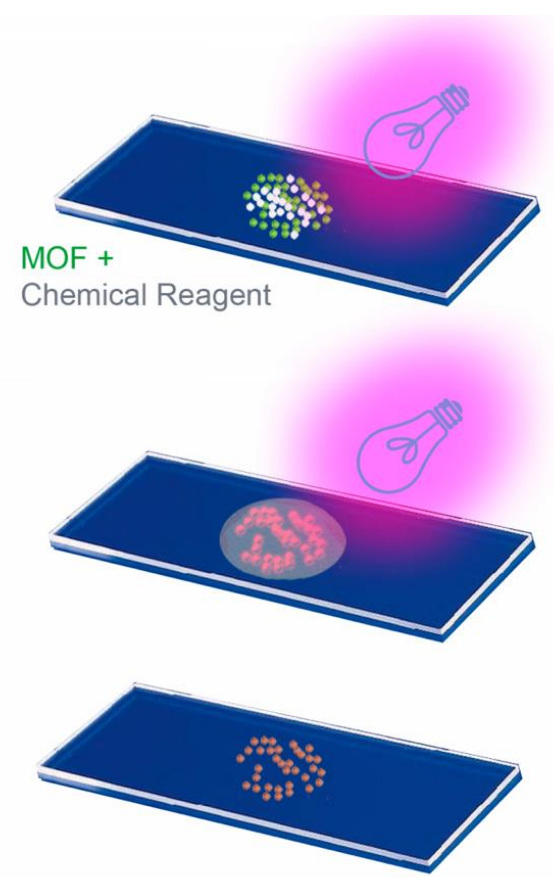

Scheme 1. Illustration of the photo-mediated CPSM of MOFs. Once irradiated with a UV-Vis lamp (top), a photothermally active MOF, previously mixed with another reagent, wil heat up, causing the other reagent to melt (middle). This in turn drives the reaction of the reagent with the functional groups on the pore walls of the MOF (below).
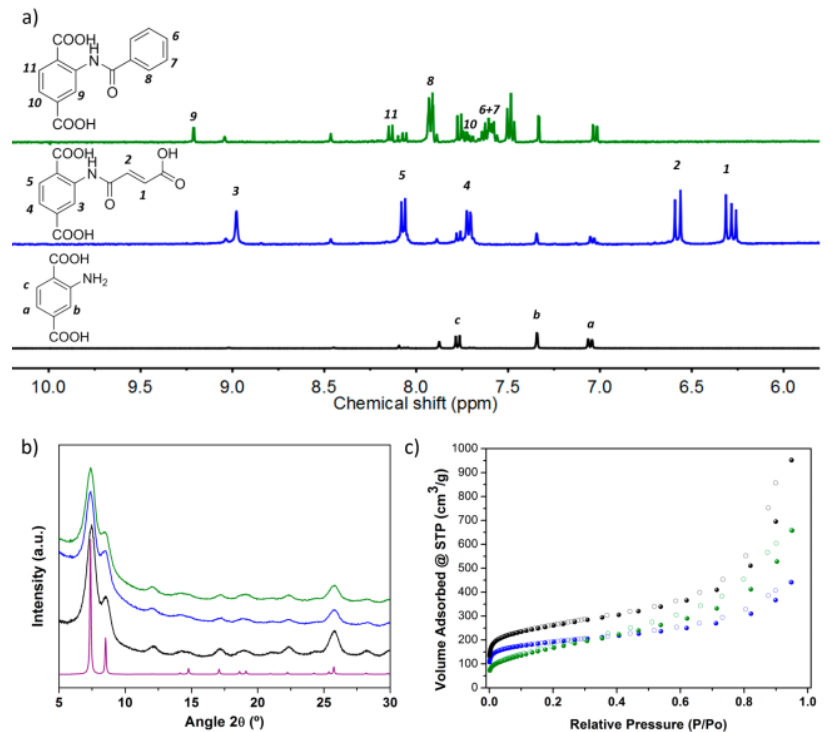

Figure 1. a) ${ }^{1} \mathrm{H}$ NMR spectra of the digested (UiO-66-MA) $)_{3}$, (blue), (UiO-66-BA) ${ }_{3}$ (green) and UiO-66- $\mathrm{NH}_{2}$ (black) in HF/DMSO- $d_{6}$. b) XRPD patterns for simulated UiO-66- $\mathrm{NH}_{2}$ (purple), activated UiO-66- $\mathrm{NH}_{2}$ (black), (UiO-66-MA) ${ }_{3}$ (blue) and (UiO66-BA) ${ }_{3}$ (green). c) $\mathrm{N}_{2}$ sorption isotherms for $\mathrm{UiO}-66-\mathrm{NH}_{2}$ (black), (UiO-66-MA) (blue) and (UiO-66-BA) 3 (green). 

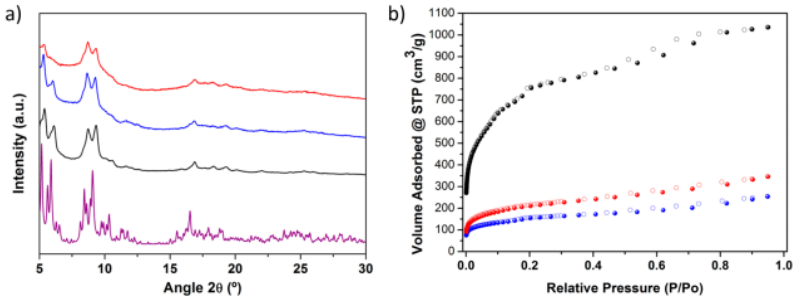

Figure 2. a) XRPD patterns for simulated MIL-101-NH2-(AI) (purple), activated MIL101-NH2-(Al) (black), (MIL-101-(Al)-MA)3 (blue) and (MIL-101-(Al)-BA)3 (red). b) N2 sorption isotherms of MIL-101-NH2-(Al) (black), (MIL-101-(Al)-MA)3 (blue) and (MIL-101-(Al)-BA)3 (red) .

Table 1. BET areas, pore volumes and conversion rates (\%) for the synthesised and modified MOFs.

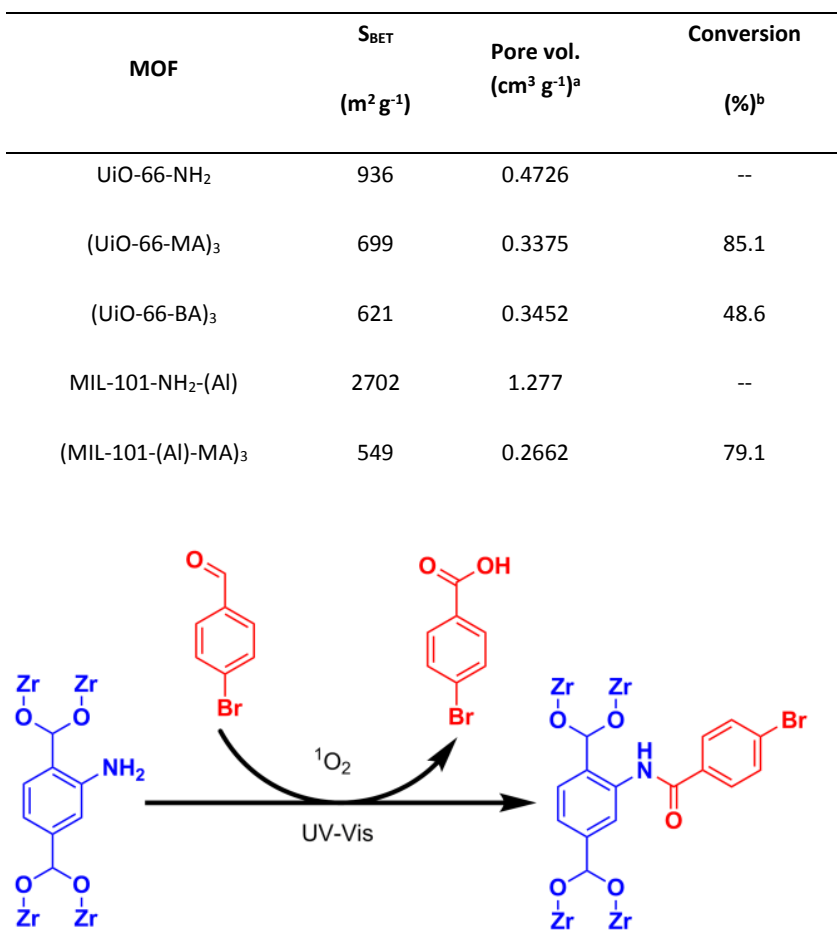

Scheme 2. Schematic representation of the CPSM reaction of UiO-66- $-\mathrm{NH}_{2}$ and $\mathrm{BrBA}$ forming an amide through a photoinduced cascade reaction. 